\section{The multiple faces of urinary glucose tetrasaccharide as biomarker for patients with hepatic glycogen storage diseases}

\section{To the Editor}

Hepatic glycogen storage diseases (GSDs) are rare inborn errors of carbohydrate metabolism. ${ }^{1}$ Clinical presentations include severe fasting intolerance, growth failure, and hepatomegaly. Biochemical characteristics are (non)ketotic hypoglycemia, hyperlactatemia, increased liver enzymes, and hyperlipidemia. Strict dietary management is the cornerstone of treatment. Routine analysis in plasma (i.e., glucose, lactate, ketones, alanine and aspartate aminotransferases [ALT and AST], creatine phosphokinase [CK], uric acid, lipids) and urine (ketones) are essential for monitoring metabolic control. ${ }^{1}$ Urinary glucose tetrasaccharide (Glc4) excretions, first described as a biomarker of GSDII (Pompe disease), can also be elevated in hepatic GSD patients. ${ }^{2}$ Indeed, in our patient cohort, urinary Glc4 excretions were increased in 10/15 GSDIa (67\%), 9/10 GSDIb (90\%), 28/28 GSDIII (100\%), 1/7 GSDIV (14\%), 3/10 GSDIX (30\%) and 8/9 GSDXI (89\%), compared with 17/22 GSDII (77\%) patient samples. Highest urinary Glc4 excretions were found in GSDIII patients.

GSDIII (OMIM 232400) is caused by a deficiency of debranching enzyme activity due to biallelic pathogenic $A G L$ variants. The International Study on GSDIII described the dual phenotypes of this disease, ranging from a merely fasting intolerance associated liver disease in childhood to a chronic, progressive muscle disease during adulthood in an important subset of the patients, in whom also heart, skeletal muscle, and bones can be affected. ${ }^{3}$ In a recent issue of Genetics in Medicine, the authors of "Liver fibrosis during clinical ascertainment of glycogen storage disease type III: a need for improved and systematic monitoring" described the natural history of liver disease in 26 pediatric GSDIII patients. ${ }^{4}$ In their single-center, retrospective, longitudinal study, a major observation was that elevated markers of liver injury (ALT, AST), hyperlipidemia, and urinary Glc4 in childhood tended to normalize with age, while CK activities were elevated and did not decrease with age.

In our experience, a case-oriented analysis is important, emphasized by observations in a recently diagnosed GSDIII patient, homozygous for the pathogenic c.4529dupA AGL variant. We observed decreased liver enzyme activities and urinary Glc4 excretions after initiation of dietary management (Supplemental Table 1). CK activities increased in conjunction with reaching milestones of physical development, such as walking. A good positive correlation was found between urinary Glc4 and plasma AST and ALT, but there was no (or even a slightly negative) association with plasma CK, which parallels the results of Halaby et al. ${ }^{4}$ In nine adult GSDIIIa patients, however, we found that urinary Glc4 was positively related to clinical signs of myopathy and plasma CK activities. In this cohort, three patients with CK activities below $250 \mathrm{U} / \mathrm{L}$ and six patients with CK above $750 \mathrm{U} / \mathrm{L}$ had median (range) urinary Glc4 excretions of $2.1(1.7-3.2$, ref $<1)$ and $20(16-29$, $\mathrm{ref}<1$ ) umol/mmol creatinine, respectively. Combining these observations, we therefore hypothesize that in pediatric GSDIII patients, decreasing Glc4 excretions reflect improved fasting tolerance (i.e., a liver function), whereas in adults, glc4 excretion may be associated with chronic, progressive skeletal muscle involvement.

Glc4 is a degradation product of glycogen and (other) branched chain starches, such as amylopectin, formed by the glycolytic activity of salivary and pancreatic $\alpha$-amylases and neutral $\alpha-1,4$-glucosidase activities. ${ }^{5,6} \mathrm{Glc} 4$ is associated with increased glycogen storage in both liver, as demonstrated in our patient and those of Halaby et al., ${ }^{4}$ and in muscle. ${ }^{7}$ In this respect, it is interesting to note that Glc4 is also elevated in Duchenne muscular dystrophy and muscle trauma. ${ }^{5,8}$ Glc4 may therefore be a good biomarker not only for GSD, but also for muscle disorders in general.

At the cellular level, Glc4 and other GSD biomarkers are at far biochemical distance from the enzymatic defect, and for some, including Glc4, the precise origin is yet unclear. Moreover, biomarkers may not adequately reflect the intracellular situation. In clinical care, blood or urine samples are usually obtained at relatively random moments, and the composition and the timing of the last meal versus acquiring the samples affects the results of many of these markers, including urinary Glc4. ${ }^{5}$ Prospective studies on the clinical relevance of biomarkers for GSD are therefore highly welcomed and were recently prioritized. ${ }^{9}$ It is important to clearly define biomarker use in different stages of the disease of interest: (1) identify the risk of developing an illness, (2) screen for subclinical disease, (3) diagnose disease, (4) categorize disease severity, and (5) predict prognosis. Metabolomics may allow us to study and identify new biomarkers for GSD patients, whereas stable isotope studies may provide a better insight in metabolic fluxes in individual patients. These studies are particularly important in the light of upcoming trials with novel therapies, including messenger RNA (mRNA) treatment and gene therapy. 


\section{SUPPLEMENTARY INFORMATION}

The online version of this article (https://doi.org/10.1038/s41436020-0878-2) contains supplementary material, which is available to authorized users.

\section{DISCLOSURE}

M.R.H.-F. is employed by an academic institute, which implies unmentioned potential conflicts of interest; is one of the principal investigators (PIs) in investigator-initiated research, for which the University Medical Center Groningen (UMCG) has received a research grant by Alfasigma Nederland BV, Nutricia, DSM, the Dutch PKU Society, and Roche Diagnostics; and has received a travel fee from the European Society for Phenylketonuria and Allied Disorders Treated as Phenylketonuria (ESPKU). All payments are to the UMCG, not to the author. F.d.B. is employed by an academic institute, which implies unmentioned potential conflicts of interest, and is investigator in the following sponsor-initiated clinical trials, for which the UMCG has received grants/research support: NCT02318966-Glycosade v UCCS in the Dietary Management of Hepatic GSD (Glyde) sponsored by Vitaflo International, Ltd. T.G.J.D. is employed by an academic institute, which implies unmentioned potential conflicts of interest; has confidentiality agreements with several pharmaceutical and medical food companies; is investigator in the following sponsorinitiated clinical trials, for which the UMCG has received grants/ research support: NCT03517085-Safety and Dose-Finding Study of DTX401 (AAV8G6PC) in Adults With Glycogen Storage Disease Type la (GSDla) sponsored by Ultragenyx Pharmaceutical Inc. and NCT02318966-Glycosade v UCCS in the Dietary Management of Hepatic GSD (Glyde) sponsored by Vitaflo International, Ltd; is a $\mathrm{PI}$ in investigator-initiated research, for which the UMCG has received a research grant by Alfasigma Nederland BV; and has performed consultations for Danone, Sigma-Tau BV, Dimension Therapeutics, Inc, and Ultragenyx Pharmaceutical Inc. for which the UMCG has been reimbursed. All contracts and agreements with private parties are via the UMCG Contract Research Desk and all payments are to the UMCG. The other authors declare no conflicts of interest.

Publisher's note Springer Nature remains neutral with regard to jurisdictional claims in published maps and institutional affiliations.

\section{Rebecca Heiner-Fokkema, PhD (iD ${ }^{1}$, Jennifer van der Krogt, BSc ${ }^{1}$, Foekje de Boer, $R D^{2}$, Marieke J. Fokkert-Wilts, $R D^{2}$,}

Ronald G. H. J. Maatman, $P h D^{1}$, Irene J. Hoogeveen, $M D^{2}$ and Terry G. J. Derks, $M D, P h D^{2}$

${ }^{1}$ Department of Laboratory Medicine, University of Groningen, University Medical Center Groningen, Groningen, The Netherlands; ${ }^{2}$ Beatrix Children's Hospital, Division of Metabolic Diseases, University of Groningen, University Medical Center Groningen, Groningen, The Netherlands. Correspondence: M. Rebecca Heiner-Fokkema (m.r.heiner@umcg.nl)

\section{REFERENCES}

1. Weinstein DA, Steuerwald U, De Souza CFM, Derks TGJ. Inborn errors of metabolism with hypoglycemia: glycogen storage diseases and inherited disorders of gluconeogenesis. Pediatr Clin North Am. 2018;65:247-265.

2. Sluiter W, Van Den Bosch JC, Goudriaan DA, et al. Rapid ultraperformance liquid chromatography-tandem mass spectrometry assay for a characteristic glycogen-derived tetrasaccharide in pompe disease and other glycogen storage diseases. Clin Chem. 2012;58:1139-1147.

3. Sentner CP, Hoogeveen IJ, Weinstein DA, et al. Glycogen storage disease type III: diagnosis, genotype, management, clinical course and outcome. J Inherit Metab Dis. 2016;39:697-704.

4. Halaby CA, Young SP, Austin S, et al. Liver fibrosis during clinical ascertainment of glycogen storage disease type III: a need for improved and systematic monitoring. Genet Med. 2019;21:2686-2694.

5. Kumlien J, Chester MA, Lindberg BS, Pizzo P, Zopf D, Lundblad A. Urinary excretion of a glucose-containing tetrasaccharide. A parameter for increased degradation of glycogen. Clin Chim Acta. 1988;176:39-48.

6. Young SP, Piraud M, Goldstein JL, et al. Assessing disease severity in Pompe disease: the roles of a urinary glucose tetrasaccharide biomarker and imaging techniques. Am J Med Genet Part C Semin Med Genet. 2012;160 C:50-58.

7. Young SP, Zhang H, Corzo D, et al. Long-term monitoring of patients with infantile-onset Pompe disease on enzyme replacement therapy using a urinary glucose tetrasaccharide biomarker. Genet Med. 2009;11:536-541.

8. Lundblad A, Svensson S, Yamashina I, Mitsuhiro O. Increased urinary excretion of a glucose-containing tetrasaccharide in patients with Duchenne muscular dystrophy. FEBS Lett. 1979;97:249-252.

9. Peeks F, Boonstra WF, de Baere $L$, et al. Research priorities for liver glycogen storage disease: an international priority setting partnership with the James Lind Alliance. J Inherit Metab Dis. 2020;43:279-289.

Open Access This article is licensed under a Creative Commons (cc) At
License, which permits any non-commercial use, sharing, distribution and reproduction in any medium or format, as long as you give appropriate credit to the original author(s) and the source, and provide a link to the Creative Commons license. You do not have permission under this license to share adapted material derived from this article or parts of it. The images or other third party material in this article are included in the article's Creative Commons license, unless indicated otherwise in a credit line to the material. If material is not included in the article's Creative Commons license and your intended use is not permitted by statutory regulation or exceeds the permitted use, you will need to obtain permission directly from the copyright holder. To view a copy of this license, visit http://creativecommons.org/licenses/by-nc-nd/4.0/.

(C) The Author(s) 2020

Advance online publication 13 July 2020. doi:10.1038/s41436-020-0878-2 